\title{
Second order modular forms
}

\author{
by \\ G. Chinta (Providence, RI), N. Diamantis (Hamilton) \\ and C. O'Sullivan (Bronx, NY)
}

1. Introduction. In some recent papers (cf. [G2], $[\mathrm{O}],[\mathrm{CG}],[\mathrm{GG}]$, [DO]) the properties of new types of Eisenstein series are investigated. Motivated by the $a b c$ conjecture, these series were originally introduced by Goldfeld ([G1], [G3]) in order to study the distribution of modular symbols. Let $f(z)$ be a fixed cusp form of weight 2 for $\Gamma=\Gamma_{0}(N)$, say, the Hecke congruence group of level $N$. Then the defining formula for the series is

$$
E^{*}(z, s)=\sum_{\tau \in \Gamma_{\infty} \backslash \Gamma}\langle\tau, f\rangle \operatorname{Im}(\tau z)^{s},
$$

for $z$ in the upper half-plane $\mathfrak{H}$ and complex $s$ with $\operatorname{Re}(s)>2$. Here

$$
\Gamma_{\infty}=\left\{\left(\begin{array}{cc}
1 & m \\
0 & 1
\end{array}\right): m \in \mathbb{Z}\right\}
$$

is the stabilizer of the cusp $\infty$ and

$$
\langle\tau, f\rangle=\int_{w_{0}}^{\tau w_{0}} f(w) d w
$$

is called a modular symbol. Its definition is independent of $w_{0}$ in $\mathfrak{H}^{*}=$ $\mathfrak{H} \cup \mathbb{Q} \cup\{i \infty\}$. The function $E^{*}$ satisfies the equation

$$
E^{*}(\gamma z, s)=E^{*}(z, s)-\langle\gamma, f\rangle E(z, s) \quad \text { for all } \gamma \in \Gamma
$$

where

$$
E(z, s)=\sum_{\tau \in \Gamma_{\infty} \backslash \Gamma} \operatorname{Im}(\tau z)^{s},
$$

the usual Eisenstein series, satisfies $E(\gamma z, s)=E(z, s)$.

From equation (1.2) and the above it is clear that

$$
E^{*}(\gamma \delta z, s)-E^{*}(\gamma z, s)-E^{*}(\delta z, s)+E^{*}(z, s)=0 \quad \text { for all } \gamma, \delta \in \Gamma \text {. }
$$

2000 Mathematics Subject Classification: 11F11, 11F25, 11F37, 82B43. 
The form of this equation motivated us to study functions with similar transformation properties.

We will work more generally with $\Gamma \subset \mathrm{PSL}_{2}(\mathbb{R})$ a Fuchsian group of the first kind. See the explanation of these in Section 2.3 of $[\mathrm{I}]$. As described there we may choose a fundamental polygon $\mathcal{F}$ to represent $\Gamma \backslash \mathfrak{H}$. We are primarily interested in groups $\Gamma$ that contain parabolic elements. The surface $\Gamma \backslash \mathfrak{H}$ will therefore not be compact and $\overline{\mathcal{F}} \cap \widehat{\mathbb{R}}$ will be a finite set of inequivalent cusps $\mathfrak{a}, \mathfrak{b}, \ldots$ for $\widehat{\mathbb{R}}=\mathbb{R} \cup\{\infty\}$. For each cusp $\mathfrak{a}$ we may choose a scaling matrix $\sigma_{\mathfrak{a}} \in \mathrm{SL}_{2}(\mathbb{R})$ that maps the upper part of the strip $\mathcal{F}_{\infty}=\{z \in \mathfrak{H}$ : $-1 / 2 \leq \operatorname{Re}(z) \leq 1 / 2\}$ to the neighborhood of $\mathfrak{a}$ in $\mathcal{F}$ (and hence $\sigma_{\mathfrak{a}} \infty=\mathfrak{a}$ ).

Next we define the spaces of functions that we are concerned with. Let $k$ be a fixed integer. If $v$ is a character of $\Gamma$ and $F$ a function on $\mathfrak{H}$ then for all $\gamma \in \Gamma$ we set

$$
\left(\left.F\right|_{k, v} \gamma\right)(z)=v(\gamma) F(\gamma z) j(\gamma, z)^{-k}
$$

and extend the action of $\Gamma$ to $\mathbb{Z}[\Gamma]$ by linearity. Here $j\left(\left(\begin{array}{ll}* & * \\ c & d\end{array}\right), z\right)=c z+d$.

Definition I. Let $M_{k}(\Gamma, v)$ be the space of maps $f: \mathfrak{H} \rightarrow \mathbb{C}$ with the following properties:

(i) $f$ is holomorphic,

(ii) $\left.f\right|_{k, v}(\gamma-1)=0$ for all $\gamma$ in $\Gamma$ (where 1 stands for the identity matrix),

(iii) $f$ has at most polynomial growth at the cusps.

The precise meaning of (iii) is that $\left(\left.f\right|_{k, v} \sigma_{\mathfrak{a}}\right)(z) \ll \operatorname{Im}(z)^{n}$ for each cusp $\mathfrak{a}$ and some constant $n$ with $z$ in the upper part of the strip $\mathcal{F}_{\infty}$. These are the modular forms of weight $k$ and character $v$ for $\Gamma$. We denote by $\widetilde{M}_{k}(\Gamma, v)$ the space obtained by relaxing (i) to include smooth functions. The non-holomorphic Eisenstein series are examples. (In this paper $\sim$ will always signify a smooth space and its absence a holomorphic space.)

DeFinition II. In a similar manner we may define the space $\widetilde{M}_{k}^{2}(\Gamma, v)$ of maps $f: \mathfrak{H} \rightarrow \mathbb{C}$ satisfying:

(i) $f$ is smooth,

(ii) $\left.f\right|_{k, v}\left(\gamma_{a}-1\right)\left(\gamma_{b}-1\right)=0$ for all $\gamma_{a}, \gamma_{b}$ in $\Gamma$,

(iii) for each $\gamma$ in $\Gamma,\left(\left.f\right|_{k, v} \gamma\right)(z)$ has at most polynomial growth at the cusps,

(iv) $\left.f\right|_{k, v}(\pi-1)=0$ for all parabolic $\pi$ in $\Gamma$.

It can be shown that $E^{*}(z, s)$ is an example of such a function, when $k=0$. If we call the holomorphic subspace $M_{k}^{2}(\Gamma, v)$ then we have the inclusions

$$
M_{k}(\Gamma, v) \subset M_{k}^{2}(\Gamma, v) \subset \widetilde{M}_{k}^{2}(\Gamma, v), \quad \widetilde{M}_{k}(\Gamma, v) \subset \widetilde{M}_{k}^{2}(\Gamma, v) .
$$


Remarks. - Condition (iv) in the definition of $\widetilde{M}_{k}^{2}(\Gamma, v)$ was included to simplify the statements of the results and because the examples we have in mind so far satisfy it. It also ensures the existence of Fourier expansions of the functions at each cusp provided $v$ is trivial on the parabolic elements.

- Condition (iii) may be strengthened by replacing polynomial growth with exponential decay,

$$
\left(\left.f\right|_{k, v} \sigma_{\mathfrak{a}}\right)(z) \ll e^{-c \operatorname{Im}(z)}
$$

for each cusp $\mathfrak{a}$ and some constant $c>0$ with, as before, $z$ in the upper part of the strip $\mathcal{F}_{\infty}$. We obtain (in an obvious notation) the spaces of smooth functions $\widetilde{S}_{k}(\Gamma, v), \widetilde{S}_{k}^{2}(\Gamma, v)$ and their holomorphic versions $S_{k}(\Gamma, v)$ and $S_{k}^{2}(\Gamma, v)$.

- It is also interesting to consider other spaces, for example smooth functions $f$ such that $\left.f\right|_{k, v}(\gamma-1) \in M_{k}(\Gamma, v)$.

We call elements of $M_{k}^{2}(\Gamma, v)$ or $\widetilde{M}_{k}^{2}(\Gamma, v)$ second-order modular forms and elements of $S_{k}^{2}(\Gamma, v)$ or $\widetilde{S}_{k}^{2}(\Gamma, v)$ second-order cuspforms. The names were suggested by D. Zagier in whose work with P. Kleban on percolation theory such functions also appear.

In this paper we show that these functions are much more basic in terms of the usual modular forms than one might think at first. In fact, their role is analogous to that of Eichler integrals with respect to period polynomials.

Another reason for the interest of second order modular forms is that the action $\left.\right|_{k, v}$ induces a natural representation of the abelianization of $\Gamma$ in $\widetilde{M}_{k}^{2}(\Gamma, v)$. Indeed, let $\varrho: \Gamma \rightarrow \operatorname{End}\left(\widetilde{M}_{k}^{2}(\Gamma, v)\right)$ be such that $\varrho(\gamma)(f)=\left.f\right|_{k, v} \gamma$ for all $\gamma \in \Gamma, f \in \widetilde{M}_{k}^{2}(\Gamma, v)$. By definition, $\left.f\right|_{k, v} \gamma \delta=\left.f\right|_{k, v} \gamma+\left.f\right|_{k, v} \delta-f$, so

$$
\left.f\right|_{k, v} \gamma \delta(\delta \gamma)^{-1}=\left.f\right|_{k, v}(\gamma+\delta-1)(\delta \gamma)^{-1}=\left.f\right|_{k, v} \delta \gamma(\delta \gamma)^{-1}=f
$$

for all $\gamma, \delta \in \Gamma, f \in \widetilde{M}_{k}^{2}(\Gamma, v)$. Similarly for the other spaces of second-order modular forms we will examine. Thanks to our work in Section 2 we can then associate such a representation to a usual modular form.

The paper is organized as follows. In Section 2 we determine the structure of the spaces $\widetilde{M}_{k}^{2}(\Gamma, v)$ and $\widetilde{S}_{k}^{2}(\Gamma, v)$. For example, if $g$ is the genus of $\Gamma \backslash \mathfrak{H}$ then an easy to state corollary of the more precise Theorem 2.3 is

Corollary 2.4. As $\mathbb{R}$-vector spaces we have

$$
\widetilde{M}_{k}^{2}(\Gamma, v) \cong \bigoplus_{i=0}^{2 g} \widetilde{M}_{k}(\Gamma, v) .
$$

Then we turn to functions that satisfy the more general equation

$$
\left.f\right|_{k, v}(\gamma-1)(\delta-1)(\varepsilon-1)=0 \quad \text { for all } \gamma, \delta, \varepsilon \in \Gamma
$$


rather than $\left.f\right|_{k, v}(\gamma-1)(\delta-1)=0(\gamma, \delta \in \Gamma)$. If the space of such functions for which $\left.f\right|_{k, v}(\gamma \delta-\delta \gamma)=0$ is called $\widetilde{M}_{k}^{3}(\Gamma, v)_{\text {ab }}$ then a consequence of Theorem 2.5 is that

$$
\widetilde{M}_{k}^{3}(\Gamma, v)_{\mathrm{ab}} \cong_{\mathbb{R}} \bigoplus_{i=1}^{(2 g+1)(g+1)} \widetilde{M}_{k}(\Gamma, v) .
$$

Furthermore, we give a partial description of the class of functions $f$ such that

$$
\left.f\right|_{k, v} p(\gamma, \ldots)=0
$$

where $p$ is an arbitrary polynomial in $\mathbb{Z}\left[x_{1}, \ldots, x_{n}\right]$ with $x_{i}$ non-commuting variables.

In Section 3 we give an analogous treatment of the subspace of secondorder modular forms that are also eigenfunctions of the Laplacian for a particular eigenvalue. These second-order Maass forms arise as residues of the function

$$
E^{*}(z, s)=\sum_{\tau \in \Gamma_{\infty} \backslash \Gamma}|\langle\tau, f\rangle|^{2} \operatorname{Im}(\tau z)^{s}
$$

studied in [G2] for example. It is hoped that a deeper understanding of these residues will help establish new results about the distribution of modular symbols.

Finally in Section 4 we show that there is a natural extension of the definition of Hecke operators that applies to second-order modular forms. These Hecke operators have the same multiplicativity and commutativity properties as the usual Hecke operators and hence the Fourier coefficients of their eigenfunctions have multiplicativity properties analogous to those of the usual Hecke eigenforms.

2. The structure of $\widetilde{M}_{k}^{2}(\Gamma, v)$. To obtain a description of the structure of $\widetilde{M}_{k}^{2}(\Gamma, v)$ we use the set of generators of $\Gamma$ given by Fricke and Klein in, say, [I]. Specifically, if $\Gamma \backslash \mathfrak{H}$ has genus $g, r$ elliptic fixed points and $m$ cusps, then there are $2 g$ hyperbolic elements $\gamma_{i}, r$ elliptic elements $\varepsilon_{i}$ and $m$ parabolic elements $\pi_{i}$ generating $\Gamma$. Furthermore, these generators satisfy the $r+1$ relations:

$$
\left[\gamma_{1}, \gamma_{g+1}\right] \ldots\left[\gamma_{g}, \gamma_{2 g}\right] \varepsilon_{1} \ldots \varepsilon_{r} \pi_{1} \ldots \pi_{m}=1, \quad \varepsilon_{j}^{e_{j}}=1
$$

for $1 \leq j \leq r$ and integers $e_{j} \geq 2$. Here $[a, b]$ denotes the commutator $a b a^{-1} b^{-1}$ of $a$ and $b$.

Recall the definition of the modular symbol $\langle\cdot, \cdot\rangle: \Gamma \times M_{2}(\Gamma) \rightarrow \mathbb{C}$ given by formula (1.1). If we take $f_{1}$ in $M_{2}(\Gamma)$ and $f_{2}$ in $S_{2}(\Gamma)$ then the map $L_{f_{1}, f_{2}}: \Gamma \rightarrow \mathbb{C}$ with

$$
L_{f_{1}, f_{2}}(\gamma)=\left\langle\gamma, f_{1}+f_{2}\right\rangle+\overline{\left\langle\gamma, f_{1}-f_{2}\right\rangle}
$$


is an element of $\operatorname{Hom}(\Gamma, \mathbb{C})$. The Eichler-Shimura isomorphism theorem for weight 2 (see, for instance, [S, Ch. 8]) states that the map $\left(f_{1}, f_{2}\right) \mapsto L_{f_{1}, f_{2}}$ is actually an $\mathbb{R}$-vector space isomorphism:

$$
M_{2}(\Gamma) \oplus S_{2}(\Gamma) \cong \operatorname{Hom}(\Gamma, \mathbb{C}) .
$$

Also if we are only interested in homomorphisms $\Gamma \rightarrow \mathbb{C}$ that are zero on the parabolic elements (call this space $\operatorname{Hom}_{0}(\Gamma, \mathbb{C})$ ) then the same map gives

$$
S_{2}(\Gamma) \oplus S_{2}(\Gamma) \cong \operatorname{Hom}_{0}(\Gamma, \mathbb{C}) .
$$

In particular for any $L$ in $\operatorname{Hom}_{0}(\Gamma, \mathbb{C})$ there exist $f, g$ in $S_{2}(\Gamma)$ so that if we define

$$
\Lambda(z):=\int_{i \infty}^{z} f(w) d w+\overline{\int_{i \infty}^{z} g(w) d w}
$$

then $L(\gamma)=\Lambda(\gamma z)-\Lambda(z)$ for all $\gamma$ in $\Gamma$ and all $z$ in $\mathfrak{H}^{*}$. With the $2 g$ hyperbolic generators $\gamma_{i}$ we next define corresponding homomorphisms $L_{i}$ such that $L_{i}\left(\gamma_{i}\right)=1$ and $L_{i}(\gamma)=0$ for all other generators $\gamma$ of $\Gamma$. Since $L_{i} \in$ $\operatorname{Hom}_{0}(\Gamma, \mathbb{C})$, there exist cusp forms $f_{i}, g_{i}$ such that $L_{i}(\gamma)=\Lambda_{i}(\gamma z)-\Lambda_{i}(z)$ with

$$
\Lambda_{i}(z):=\int_{i \infty}^{z} f_{i}(w) d w+\overline{\int_{i \infty}^{z} g_{i}(w) d w .}
$$

Lemma 2.1. For each $\gamma \in \Gamma$ we have the map $\left.f \mapsto f\right|_{k, v}(\gamma-1)$. This map sends $\widetilde{M}_{k}^{2}(\Gamma, v)$ to $\widetilde{M}_{k}(\Gamma, v)$.

The proof follows directly from the definitions of these spaces. In a similar manner these maps send

$$
M_{k}^{2}(\Gamma, v) \rightarrow M_{k}(\Gamma, v), \quad \widetilde{S}_{k}^{2}(\Gamma, v) \rightarrow \widetilde{S}_{k}(\Gamma, v), \quad S_{k}^{2}(\Gamma, v) \rightarrow S_{k}(\Gamma, v) .
$$

Lemma 2.2. For $f$ a second-order modular form we have $f_{k, v}(\varepsilon-1)=0$ for all elliptic elements of $\Gamma$.

Proof. If $\varepsilon^{n}=1$ then

$$
\begin{aligned}
\left.f\right|_{k, v}(\varepsilon-1) & =\left.f\right|_{k, v}\left(\varepsilon^{n+1}-1\right)=\left.f\right|_{k, v}(\varepsilon-1)\left(1+\varepsilon+\varepsilon^{2}+\ldots+\varepsilon^{n}\right) \\
& =\left.(n+1) f\right|_{k, v}(\varepsilon-1) .
\end{aligned}
$$

Therefore $\left.n f\right|_{k, v}(\varepsilon-1)=0$ and the lemma is proved.

Theorem 2.3 (Chinese Remainder Theorem). For $f$ in $\widetilde{M}_{k}^{2}(\Gamma, v)$ we let $\psi$ denote the map sending $f$ to the vector $\left(\left.f\right|_{k, v}\left(\gamma_{1}-1\right), \ldots,\left.f\right|_{k, v}\left(\gamma_{2 g}-1\right)\right)$. Then the following sequence of maps is exact:

$$
0 \rightarrow \widetilde{M}_{k}(\Gamma, v) \hookrightarrow \widetilde{M}_{k}^{2}(\Gamma, v) \stackrel{\psi}{\rightarrow} \bigoplus_{i=1}^{2 g} \widetilde{M}_{k}(\Gamma, v) \rightarrow 0 .
$$


In other words, for each set $\left\{f_{i}: i=1, \ldots, 2 g\right\} \subset \widetilde{M}_{k}(\Gamma, v)^{2 g}$ there is a $h \in \widetilde{M}_{k}^{2}(\Gamma, v)$ (unique up to addition by a form in $\widetilde{M}_{k}(\Gamma, v)$ ) such that $f_{i}=\left.h\right|_{k, v}\left(\gamma_{i}-1\right)(i=1, \ldots, 2 g)$, and conversely.

Proof. To prove the exactness of the middle term we observe that if $f$ is in the kernel of $\psi$ then we must have $\left.f\right|_{k, v}(\gamma-1)=0$ for all $\gamma$ in $\Gamma$ since it is true for each of the parabolic, elliptic and hyperbolic generators of the group. Thus $\operatorname{Ker}(\psi)=\widetilde{M}_{k}(\Gamma, v)$.

Finally, to prove that $\psi$ is surjective we note that for any vector $V=$ $\left(f_{1}, \ldots, f_{2 g}\right)$ in $\bigoplus_{i=1}^{2 g} \widetilde{M}_{k}(\Gamma, v)$ we have $\psi\left(\sum_{i=1}^{2 g} f_{i} \Lambda_{i}\right)=V$. It is routine to check that $\sum_{i=1}^{2 g} f_{i} \Lambda_{i}$ is in $\widetilde{M}_{k}^{2}(\Gamma, v)$. This completes the proof of Theorem 2.3.

The same proof gives the exact sequence

$$
0 \rightarrow \widetilde{S}_{k}(\Gamma, v) \hookrightarrow \widetilde{S}_{k}^{2}(\Gamma, v) \stackrel{\psi}{\rightarrow} \bigoplus_{i=1}^{2 g} \widetilde{S}_{k}(\Gamma, v) \rightarrow 0 .
$$

For the holomorphic spaces $M_{k}^{2}(\Gamma, v)$ and $S_{k}^{2}(\Gamma, v)$ the above proof fails since $\Lambda_{i}(z)$ is not always holomorphic. In light of this difficulty it is natural to define the hybrid subspace $\widetilde{M}_{k}^{2}(\Gamma, v)^{*} \subset \widetilde{M}_{k}^{2}(\Gamma, v)$ of smooth functions that satisfy

$$
\left.f\right|_{k, v}(\gamma-1) \in M_{k}(\Gamma, v) \quad \text { for all } \gamma \in \Gamma
$$

and similarly for $\widetilde{S}_{k}^{2}(\Gamma, v)^{*}$. The proof of Theorem 2.3 then gives

$$
\begin{aligned}
& 0 \rightarrow \widetilde{M}_{k}(\Gamma, v) \hookrightarrow \widetilde{M}_{k}^{2}(\Gamma, v)^{*} \stackrel{\psi}{\rightarrow} \bigoplus_{i=1}^{2 g} M_{k}(\Gamma, v) \rightarrow 0, \\
& 0 \rightarrow \widetilde{S}_{k}(\Gamma, v) \hookrightarrow \widetilde{S}_{k}^{2}(\Gamma, v)^{*} \stackrel{\psi}{\rightarrow} \bigoplus_{i=1}^{2 g} S_{k}(\Gamma, v) \rightarrow 0 .
\end{aligned}
$$

An easy consequence of Theorem 2.3 is

COROLlary 2.4. We have the $\mathbb{R}$-vector space isomorphism

$$
\widetilde{M}_{k}^{2}(\Gamma, v) \cong \bigoplus_{i=0}^{2 g} \widetilde{M}_{k}(\Gamma, v)
$$

and for any $f \in \widetilde{M}_{k}^{2}(\Gamma, v)$ there exist unique $h_{i} \in \widetilde{M}_{k}(\Gamma, v)$ for $0 \leq i \leq 2 g$ such that

$$
f=\sum_{i=0}^{2 g} h_{i} \Lambda_{i}
$$

where the functions $\Lambda_{i}$ are as defined earlier and for convenience we set $\Lambda_{0}(z)=1$. Similar results hold for the spaces $\widetilde{S}_{k}(\Gamma, v), \widetilde{M}_{k}(\Gamma, v)^{*}$ and $\widetilde{S}_{k}(\Gamma, v)^{*}$. 
A natural generalization of second-order forms satisfying the transformation property:

$$
\left.f\right|_{k, v}\left(\gamma_{a}-1\right)\left(\gamma_{b}-1\right)=0 \quad \text { for all } \gamma_{a}, \gamma_{b} \in \Gamma
$$

in Definition II would be functions satisfying the new condition (ii),

$$
\left.f\right|_{k, v}\left(\gamma_{a}-1\right)\left(\gamma_{b}-1\right)\left(\gamma_{c}-1\right)=0 \quad \text { for all } \gamma_{a}, \gamma_{b}, \gamma_{c} \in \Gamma .
$$

We might call such functions third-order modular forms and in a consistent notation write $\widetilde{M}_{k}^{3}(\Gamma, v), \widetilde{S}_{k}^{3}(\Gamma, v)$, etc.

We may characterize third-order modular forms in an analogous way to Theorem 2.3 but there is an important difference. While it was true for $f$ in $\widetilde{M}_{k}(\Gamma, v)$ or $\widetilde{M}_{k}^{2}(\Gamma, v)$ that $\left.f\right|_{k, v}\left(\gamma_{a} \gamma_{b}-\gamma_{b} \gamma_{a}\right)=0$ this is no longer necessarily the case for third-order modular forms.

If $f \in \widetilde{M}_{k}^{3}(\Gamma, v)$ then it is easy to check that the analogs of Lemmas 2.1 and 2.2 are true. In other words $\widetilde{M}_{k}^{3}(\Gamma, v) \rightarrow \widetilde{M}_{k}^{2}(\Gamma, v)$ under the map $f \mapsto$ $\left.f\right|_{k, v}(\gamma-1)$ for each $\gamma \in \Gamma$ and $\left.f\right|_{k, v}(\varepsilon-1)=0$ for all elliptic $\varepsilon \in \Gamma$. Define the map

$$
\psi^{*}: \widetilde{M}_{k}^{3}(\Gamma, v) \rightarrow \bigoplus_{i=1}^{(2 g)^{2}} \widetilde{M}_{k}(\Gamma, v)
$$

with

$$
\psi^{*}(f)=\left(\left.f\right|_{k, v}\left(\gamma_{i}-1\right)\left(\gamma_{j}-1\right)\right)_{1 \leq i, j \leq 2 g} .
$$

Set $\delta_{i j}=1$ if $i=j$ and zero otherwise. If there exist smooth functions $\Lambda_{i j}(z)$ (with at most polynomial growth at the cusps) satisfying, for $1 \leq i, j, m, n$ $\leq 2 g$,

$$
\Lambda_{i j}\left(\gamma_{m} \gamma_{n} z\right)-\Lambda_{i j}\left(\gamma_{m} z\right)-\Lambda_{i j}\left(\gamma_{n} z\right)+\Lambda_{i j}(z)=\delta_{i m} \delta_{j n}
$$

then

$$
0 \rightarrow \widetilde{M}_{k}^{2}(\Gamma, v) \hookrightarrow \widetilde{M}_{k}^{3}(\Gamma, v) \stackrel{\psi^{*}}{\rightarrow} \bigoplus_{i=1}^{(2 g)^{2}} \widetilde{M}_{k}(\Gamma, v) \rightarrow 0
$$

by essentially the same proof as Theorem 2.3 and

$$
f=\sum_{i=0}^{2 g} h_{i} \Lambda_{i}+\sum_{1 \leq i, j \leq 2 g} h_{i j} \Lambda_{i j}
$$

for unique $h_{i}, h_{i j} \in \widetilde{M}_{k}(\Gamma, v)$. Unfortunately the functions $\Lambda_{i j}$ satisfying (2.3) remain to be found. Without them the above results are not valid.

The products $\Lambda_{i}(z) \Lambda_{j}(z)$ are very close to satisfying (2.3). Their only defect is that they fail to distinguish between $\gamma_{a} \gamma_{b}$ and $\gamma_{b} \gamma_{a}$. They do allow us to prove the following

THEOREM 2.5. Let $\widetilde{M}_{k}^{3}(\Gamma, v)_{\mathrm{ab}} \subset \widetilde{M}_{k}^{3}(\Gamma, v)$ denote the subspace of third-order modular forms $f$ that satisfy the additional abelian condition 


$$
\begin{aligned}
& \left.f\right|_{k, v}\left(\gamma_{a} \gamma_{b}\right)=\left.f\right|_{k, v}\left(\gamma_{b} \gamma_{a}\right) \text { for all } \gamma_{a}, \gamma_{b} \in \Gamma \text {. Then } \\
& \qquad f=\sum_{i=0}^{2 g} \sum_{j=i}^{2 g} h_{i j} \Lambda_{i} \Lambda_{j} \quad \text { for unique } h_{i j} \in \widetilde{M}_{k}(\Gamma, v) .
\end{aligned}
$$

Proof. This theorem follows from our above discussion and the formula

$$
\begin{array}{r}
\Lambda_{i}\left(\gamma_{m} \gamma_{n} z\right) \Lambda_{j}\left(\gamma_{m} \gamma_{n} z\right)-\Lambda_{i}\left(\gamma_{m} z\right) \Lambda_{j}\left(\gamma_{m} z\right)-\Lambda_{i}\left(\gamma_{n} z\right) \Lambda_{j}\left(\gamma_{n} z\right)+\Lambda_{i}(z) \Lambda_{j}(z) \\
=\left.\Lambda_{i} \Lambda_{j}\right|_{0,1}\left(\gamma_{m}-1\right)\left(\gamma_{n}-1\right)=\delta_{i m} \delta_{j n}+\delta_{j m} \delta_{i n} .
\end{array}
$$

To prove this formula we write

$$
\begin{aligned}
& \Lambda_{i}\left(\gamma_{m} z\right) \Lambda_{j}\left(\gamma_{m} z\right)-\Lambda_{i}(z) \Lambda_{j}(z) \\
& \quad=\Lambda_{i}\left(\gamma_{m} z\right)\left(\Lambda_{j}\left(\gamma_{m} z\right)-\Lambda_{j}(z)\right)+\left(\Lambda_{i}\left(\gamma_{m} z\right)-\Lambda_{i}(z)\right) \Lambda_{j}(z) \\
& \quad=\delta_{j m} \Lambda_{i}\left(\gamma_{m} z\right)+\delta_{i m} \Lambda_{j}(z)
\end{aligned}
$$

and so

$$
\begin{aligned}
\delta_{j m} \Lambda_{i}\left(\gamma_{m} \gamma_{n} z\right) & +\delta_{i m} \Lambda_{j}\left(\gamma_{n} z\right)-\delta_{j m} \Lambda_{i}\left(\gamma_{m} z\right)-\delta_{i m} \Lambda_{j}(z) \\
& =\delta_{j m}\left(\Lambda_{i}\left(\gamma_{m} \gamma_{n} z\right)-\Lambda_{i}\left(\gamma_{m} z\right)\right)+\delta_{i m}\left(\Lambda_{j}\left(\gamma_{n} z\right)-\Lambda_{j}(z)\right) \\
& =\delta_{j m} \delta_{i n}+\delta_{i m} \delta_{j n}
\end{aligned}
$$

as required, completing the proof.

These ideas extend to higher order modular forms. More generally if $p$ is a fixed polynomial in $\mathbb{Z}\left[x_{1}, \ldots, x_{n}\right]$, for non-commuting variables $x_{1}, \ldots, x_{n}$, consider replacing condition (ii) in Definition II with

$$
\left.f\right|_{k, v} p\left(\gamma_{a}, \gamma_{b}, \ldots\right)=0 \quad \text { for all } \gamma_{a}, \gamma_{b}, \ldots \in \Gamma \text {. }
$$

Simple examples have $p(\gamma)=\gamma^{n}-1$ or $p\left(\gamma_{a}, \gamma_{b}\right)=\gamma_{a} \gamma_{b}-\gamma_{a}$. Label these spaces $\widetilde{M}_{k}(N, v, p), \widetilde{S}_{k}(N, v, p)$, etc. For general polynomials we cannot give a simple characterization of them. However, some simple propositions may be proved.

Proposition 2.6. For a fixed $p \in \mathbb{Z}\left[x_{1}, \ldots, x_{n}\right]$ we have $\widetilde{M}_{k}(\Gamma, v) \subset$ $\widetilde{M}_{k}(\Gamma, v, p)$ provided $\widetilde{M}_{k}(\Gamma, v, p) \neq 0$.

Proof. For every $f \in \widetilde{M}_{k}(\Gamma, v)$ we have $\left.f\right|_{k, v} \gamma=f$ for all $\gamma \in \Gamma$. Therefore

$$
\left.f\right|_{k, v} p\left(\gamma_{a}, \ldots\right)=A f
$$

where $A$ is the sum of coefficients of $p$. However, $A$ must be 0 because if $g \in \widetilde{M}_{k}(\Gamma, v, p)$ is non-zero, then $\left.g\right|_{k, v} p\left(\gamma_{a}, \gamma_{b}, \ldots\right)=0$ for all $\gamma_{a}, \gamma_{b}, \ldots \in \Gamma$ and, in particular, for $\gamma_{a}=\gamma_{b}=\ldots=1$, the identity in $\Gamma$.

In the opposite direction we have 
Proposition 2.7. If $p$ has exactly two terms with coefficients summing to zero then there exists a subgroup $\Gamma_{p}$ of $\Gamma$ such that $\left.f\right|_{k, v} p\left(\gamma_{a}, \gamma_{b}, \ldots\right)=0$ for all $\gamma_{a}, \gamma_{b}, \ldots$ in $\Gamma$ if and only if $\left.f\right|_{k, v}(\gamma-1)=0$ for all $\gamma$ in $\Gamma_{p}$.

Proof. The polynomial $p$ has the form $n \delta_{1}-n \delta_{2}$ with $n$ in $\mathbb{Z}$ and $\delta_{1}, \delta_{2}$ made up of combinations of elements of $\Gamma$. Clearly we may replace $p$ by $\delta_{1}-\delta_{2}$. Also replacing $z$ by $\delta_{2}^{-1} z$ we see that the functions $f$ must satisfy $\left.f\right|_{k, v}\left(\delta_{1} \delta_{2}^{-1}-1\right)=0$. This is equivalent to $\left.f\right|_{k, v}(\gamma-1)=0$ for all $\gamma$ in the group generated by elements of the form $\delta_{1} \delta_{2}^{-1}$ since if $\left.f\right|_{k, v}\left(\gamma_{a}-1\right)=0$ and $\left.f\right|_{k, v}\left(\gamma_{b}-1\right)=0$ then

$$
\left.f\right|_{k, v}\left(\gamma_{a} \gamma_{b}-1\right)=\left.f\right|_{k, v}\left(\left(\gamma_{a}-1\right) \gamma_{b}+\left(\gamma_{b}-1\right)\right)=0 .
$$

This completes the proof.

It would be interesting to characterize $\widetilde{M}_{k}(\Gamma, v, p)$ when $p$ is a more complicated polynomial, for example

$$
p\left(\gamma_{a}, \gamma_{b}\right)=\left(\gamma_{a}-1\right)\left(\gamma_{b}-1\right)+\left(\gamma_{b}-1\right)\left(\gamma_{a}-1\right) .
$$

3. Second-order Maass cusp forms. For simplicity in the following we restrict ourselves to the case $k=0$ and $v \equiv 1$, so we write | rather than $\left.\right|_{0, v}$. In this situation we shall call $\widetilde{S}_{0}^{2}(\Gamma, 1)$ simply $\mathcal{A}^{2}(\Gamma \backslash \mathfrak{H})$. Let $\Delta=-4 y^{2} \partial_{\bar{z}} \partial_{z}$ be the hyperbolic Laplace operator. We call a function $f$ in $\mathcal{A}^{2}(\Gamma \backslash \mathfrak{H})$ a second-order Maass forms with eigenvalue $\lambda$ if $(\Delta+\lambda) f=0$. The set of all such functions we denote by $\mathcal{A}_{\lambda}^{2}(\Gamma \backslash \mathfrak{H})$. Condition (iv) in the definition of $\widetilde{S}_{0}^{2}(\Gamma, 1)$ implies that any member $f$ has a Fourier expansion at every cusp. We call $f$ a second-order Maass cuspform if the constant coefficient of $f$ at each cusp is identically zero. Denote the space of second-order Maass cuspforms of eigenvalue $\lambda$ by $\mathcal{C}_{\lambda}^{2}(\Gamma \backslash \mathfrak{H})$.

To determine the structure of $\mathcal{C}_{\lambda}^{2}(\Gamma \backslash \mathfrak{H})$ we first fix some notation. We let $\mathcal{L}(\Gamma \backslash \mathfrak{H})$ denote the space of automorphic functions on $\Gamma \backslash \mathfrak{H}$ which are square-integrable with respect to the measure

$$
d \mu(z)=\frac{d x d y}{y^{2}}, \quad z=x+i y .
$$

The subspace of automorphic eigenfuctions of the Laplacian with eigenvalue $\lambda$ is denoted by $\mathcal{C}_{\lambda}(\Gamma \backslash \mathfrak{H})$. We also fix orthonormal eigenbases $\left\{g_{i}\right\}$ and $\left\{u_{i}\right\}$ for $S_{2}(\Gamma)$ and $\mathcal{C}_{\lambda}(\Gamma \backslash \mathfrak{H})$, respectively. These bases are orthonormal with respect to the usual Petersson scalar product

$$
\langle g, h\rangle:=\int_{\Gamma \backslash \mathfrak{H}} y^{k} g(z) \overline{h(z)} d \mu(z)
$$

where $k$ is the weight. 
Since the hyperbolic Laplacian is $\mathrm{SL}_{2}(\mathbb{R})$-invariant, it follows that, for $f \in \mathcal{C}_{\lambda}^{2}(\Gamma \backslash \mathfrak{H})$, the function $z \mapsto f(\gamma z)-f(z)$ is in $\mathcal{C}_{\lambda}(\Gamma \backslash \mathfrak{H})$. Thus, as in the proof of surjectivity in Theorem 2.3 we can prove

Proposition 3.1. Let $f \in \mathcal{C}_{\lambda}^{2}(\Gamma \backslash \mathfrak{H})$. Then there exist complex constants $\left\{\alpha_{i j}\right\}$ and $\left\{\beta_{i j}\right\}$ such that, for all $\gamma \in \Gamma$,

$$
f(\gamma z)=f(z)+\sum_{i, j} u_{j}(z)\left(\alpha_{i j}\left\langle\gamma, g_{i}\right\rangle+\beta_{i j} \overline{\left\langle\gamma, g_{i}\right\rangle}\right) .
$$

We now give a characterization of the quotient $\mathcal{C}_{\lambda}^{2}(\Gamma \backslash \mathfrak{H}) / \mathcal{C}_{\lambda}(\Gamma \backslash \mathfrak{H})$ analogous to that given in Theorem 2.3.

Let first $f$ be an element of $\mathcal{C}_{\lambda}^{2}(\Gamma \backslash \mathfrak{H})$. If $\left\{\alpha_{i j}\right\}$ and $\left\{\beta_{i j}\right\}$ are the constants associated to $f$ by Proposition 3.1, define the function

$$
f_{0}(z):=\sum_{i, j} u_{j}(z)\left(\alpha_{i j} \int_{i \infty}^{z} g_{i}(w) d w+\beta_{i j} \overline{\left(\int_{i \infty}^{z} g_{i}(w) d w\right)}\right) .
$$

We note that, since we have fixed bases for $S_{2}(\Gamma)$ and $\mathcal{C}_{\lambda}(\Gamma \backslash \mathfrak{H})$, the function

$$
f_{0}(z)=f_{0}\left(z ; \lambda,\left\{\alpha_{i j}\right\},\left\{\beta_{i j}\right\}\right)
$$

is completely determined by the complex numbers $\left\{\alpha_{i j}\right\}$ and $\left\{\beta_{i j}\right\}$ and the eigenvalue $\lambda$.

Lemma 3.2. The function $G:=f-f_{0}$ is in $\mathcal{L}(\Gamma \backslash \mathfrak{H})$.

Proof. The automorphicity of $G$ is obvious from (3.1) and Proposition 3.1. The square integrability follows from the rapid decay of both $f$ and $f_{0}$ at the cusps. This completes the proof.

Thus we have characterized the function $f$ in $\mathcal{C}_{\lambda}^{2}(\Gamma \backslash \mathfrak{H})$ modulo the square integrable automorphic function $G$. To go further, we quickly review the spectral theory of $\mathcal{L}(\Gamma \backslash \mathfrak{H})$.

Let

$$
E_{\mathfrak{a}}(z, s)=\sum_{\gamma \in \Gamma_{\mathfrak{a}} \backslash \Gamma} \operatorname{Im}\left(\sigma_{\mathfrak{a}}^{-1} \gamma z\right)^{s}
$$

be the real analytic Eisenstein series for $\Gamma$ associated to the stabilizer $\Gamma_{\mathfrak{a}}$ of the cusp $\mathfrak{a}$. Then the spectral theorem for $\mathcal{L}(\Gamma \backslash \mathfrak{H})$ says that there exists an orthonormal set of eigenforms $\eta_{1}, \eta_{2}, \ldots$ with corresponding eigenvalues $\lambda_{1}, \lambda_{2}, \ldots$ such that any $u \in \mathcal{L}(\Gamma \backslash \mathfrak{H})$ has the decomposition

$$
\begin{aligned}
u(z)= & \frac{\langle u, 1\rangle}{\langle 1,1\rangle}+\sum_{j=1}^{\infty}\left\langle u, \eta_{j}\right\rangle \eta_{j}(z) \\
& +\frac{1}{4 \pi} \sum_{\mathfrak{a}} \int_{-\infty}^{\infty}\left\langle u, E_{\mathfrak{a}}(\cdot, 1 / 2+i r)\right\rangle E_{\mathfrak{a}}(z, 1 / 2+i r) d r
\end{aligned}
$$

where the second sum is over a set of inequivalent cusps. 
Now, with $f=f_{0}-G$ as above,

$$
\begin{aligned}
0 & =(\Delta+\lambda) f(z)=(\Delta+\lambda)\left(f_{0}-G\right)(z) \\
& =-\sum 4 y^{2}\left(\alpha_{i j} g_{i}(z) \partial_{\bar{z}} u_{j}(z)+\beta_{i j} \bar{g}_{i}(z) \partial_{z} u_{j}(z)\right)-(\Delta+\lambda) G(z) .
\end{aligned}
$$

Hence $(\Delta+\lambda) G=H$, say, where

$$
\begin{aligned}
H(z) & =H\left(z ; \lambda,\left\{\alpha_{i j}\right\},\left\{\beta_{i j}\right\}\right) \\
& =-\sum 4 y^{2}\left(\alpha_{i j} g_{i}(z) \partial_{\bar{z}} u_{j}(z)+\beta_{i j} \bar{g}_{i}(z) \partial_{z} u_{j}(z)\right) .
\end{aligned}
$$

Expressing both $G$ and $H$ in the form (3.2) and equating the coefficients, we get

$$
\left(\lambda-\lambda_{j}\right)\left\langle G, \eta_{j}\right\rangle=\left\langle H, \eta_{j}\right\rangle
$$

and

$$
\left(\lambda+\left(1 / 4+r^{2}\right)\right)\left\langle G, E_{\mathfrak{a}}(\cdot, 1 / 2+i r)\right\rangle=\left\langle H, E_{\mathfrak{a}}(\cdot, 1 / 2+i r)\right\rangle,
$$

for all $j, \mathfrak{a}$ and $r>0$. In particular,

$$
\left\langle H\left(\cdot ; \lambda,\left\{\alpha_{i j}\right\},\left\{\beta_{i j}\right\}\right), \eta\right\rangle=0 \quad \text { for all } \eta \in \mathcal{C}_{\lambda}(\Gamma \backslash \mathfrak{H})
$$

and

$$
\begin{aligned}
& \left\langle H\left(\cdot ; \lambda,\left\{\alpha_{i j}\right\},\left\{\beta_{i j}\right\}\right), E_{\mathfrak{a}}(\cdot, 1 / 2+i \kappa)\right\rangle=0 \\
& \quad \text { for all cusps } \mathfrak{a}, \lambda=-\left(1 / 4+\kappa^{2}\right) .
\end{aligned}
$$

The requirements given in (3.4) and (3.5) impose certain linear relations on the $\left\{\alpha_{i j}\right\}$ and $\left\{\beta_{i j}\right\}$ which must be satisfied.

It turns out that the relation (3.5) always holds: Then

Proposition 3.3. Let $\phi \in S_{2}(\Gamma)$ and $u \in \mathcal{C}_{\lambda}(\Gamma \backslash \mathfrak{H}), \lambda=-\left(1 / 4+\kappa^{2}\right)$.

$$
\left\langle 4 y^{2} \phi \partial_{\bar{z}} u, E_{\mathfrak{a}}(\cdot, 1 / 2+i \kappa)\right\rangle=\left\langle 4 y^{2} \bar{\phi} \partial_{z} u, E_{\mathfrak{a}}(\cdot, 1 / 2+i \kappa)\right\rangle=0
$$

Proof. Without loss of generality assume $\mathfrak{a}=i \infty$. Also choose $s$ with $\operatorname{Re}(s)>3$. Let

$$
\Phi(z):=\int_{i \infty}^{z} \phi(w) d w
$$

be an antiderivative of $\phi$. Note that $(\Delta+\lambda)(u \Phi)=-4 y^{2} \phi \partial_{\bar{z}} u$. Unfolding the integral, we obtain

$$
\begin{aligned}
-4\left\langle y^{2} \phi \partial_{\bar{z}} u, E_{\mathfrak{a}}(\cdot, \bar{s})\right\rangle & =\left\langle(\Delta+\lambda)(u \Phi), E_{\mathfrak{a}}(\cdot, \bar{s})\right\rangle \\
& =\int_{0}^{\infty} \int_{0}^{1}(\Delta+\lambda)(u(z) \Phi(z)) y^{s} \frac{d x d y}{y^{2}} .
\end{aligned}
$$


Integrate by parts twice. The fact that the real part of $s$ is sufficiently large ensures that the boundary terms vanish, leaving us with

$$
-4\left\langle y^{2} \phi \partial_{\bar{z}} u, E_{\mathfrak{a}}(\cdot, \bar{s})\right\rangle=\int_{0}^{\infty} \int_{0}^{1} u(z) \Phi(z)(\Delta+\lambda) y^{s} \frac{d x d y}{y^{2}} .
$$

The proof is completed by analytically continuing to $s=1 / 2+i \kappa$, and using the fact that $(\Delta+\lambda) y^{1 / 2+i \kappa}=0$.

Conversely, given complex constants $\left\{\alpha_{i j}\right\}$ and $\left\{\beta_{i j}\right\}$ satisfying (3.4), we can construct a function $f \in \mathcal{C}_{\lambda}^{2}(\Gamma \backslash \mathfrak{H}) / \mathcal{C}_{\lambda}(\Gamma \backslash \mathfrak{H})$. We first set $H:=(\Delta+\lambda) f_{0}$, where $f_{0}(z)$ is the function associated to $\left\{\alpha_{i j}\right\},\left\{\beta_{i j}\right\}$ by (3.1). Thanks to the relation (3.4), there exists a function $G \in \mathcal{L}(\Gamma \backslash \mathfrak{H})$ such that $H:=(\Delta+\lambda) G$. This function is well-defined modulo $\mathcal{C}_{\lambda}(\Gamma \backslash \mathfrak{H})$. It follows that $f:=f_{0}-G$ is an element of $\mathcal{C}_{\lambda}^{2}(\Gamma \backslash \mathfrak{H})$. Furthermore, any two functions $f \in \mathcal{C}_{\lambda}^{2}(\Gamma \backslash \mathfrak{H})$ associated to the complex constants $\left\{\alpha_{i j}\right\}$ and $\left\{\beta_{i j}\right\}$ as above must differ by a Maass form in $\mathcal{C}_{\lambda}(\Gamma \backslash \mathfrak{H})$.

It is easy to see that the mappings defined in this way are linear and inverse to one another.

Let $M=2 \operatorname{dim}\left(S_{2}(\Gamma)\right) \operatorname{dim}\left(\mathcal{C}_{\lambda}(\Gamma \backslash \mathfrak{H})\right)$. We have shown

Theorem 3.4. As an $\mathbb{R}$-vector space, $\mathcal{C}_{\lambda}^{2}(\Gamma \backslash \mathfrak{H}) / C_{\lambda}(\Gamma \backslash \mathfrak{H})$ is isomorphic to

$$
\left\{\left(\alpha_{i j}, \beta_{i j}\right) \in \mathbb{C}^{M}:\left\langle H\left(\cdot ; \lambda,\left\{\alpha_{i j}\right\},\left\{\beta_{i j}\right\}\right), \eta\right\rangle=0 \text { for all } \eta \in \mathcal{C}_{\lambda}(\Gamma \backslash \mathfrak{H})\right\} .
$$

In particular, $\operatorname{dim} \mathcal{C}_{\lambda}^{2}(\Gamma \backslash \mathfrak{H}) \leq\left(2 \operatorname{dim} S_{2}(\Gamma)+1\right) \operatorname{dim}\left(\mathcal{C}_{\lambda}(\Gamma \backslash \mathfrak{H})\right)$. It would be desirable to also find a strong lower bound for $\operatorname{dim} \mathcal{C}_{\lambda}^{2}(\Gamma \backslash \mathfrak{H})$.

4. Hecke operators. Using the above description of the structure of $\widetilde{M}_{k}^{2}(\Gamma, v)$, we can define operators on it that are compatible with the usual Hecke operators on $\widetilde{M}_{k}(\Gamma, v)$. We restrict ourselves to the case $\Gamma=\Gamma_{0}(N)$, the Hecke congruence group of level $N$ (where we have identified \pm 1 ). The character $v$ is induced by a character on $(\mathbb{Z} / N \mathbb{Z})^{*}$. In the following we will just indicate the level $N$ instead of writing the full group $\Gamma_{0}(N)$.

According to Section 2 , any $f$ in $\widetilde{M}_{k}^{2}(N, v)$ can be uniquely written in the form

$$
f(z)=\sum_{i=0}^{2 g} h_{i}(z) \Lambda_{i}(z)
$$

for unique $h_{i}$ in $\widetilde{M}_{k}(N, v)$. We then naturally define

$$
\left(T_{n} f\right)(z):=\sum_{i=0}^{2 g}\left(T_{n} h_{i}\right)(z) \Lambda_{i}(z)
$$


where the $T_{n}$ is the usual Hecke operator on $\widetilde{M}_{k}(N, v)$ given by the formula

$$
T_{n} g:=n^{k-1} \sum_{a d=n} \sum_{b(\bmod d)} v(d) d^{-k} g\left(\frac{a z+b}{d}\right) .
$$

Obviously the maps given by (4.1) map $\widetilde{M}_{k}^{2}(N, v)$ to $\widetilde{M}_{k}^{2}(N, v)$ and coincide with the usual Hecke operators on $\widetilde{M}_{k}(N, v) \subset M_{k}^{2}(N, v)$.

It is possible to define other Hecke operators on these second-order spaces. For examples of such alternative operators in the special case of Eisenstein series formed with modular symbols and related functions, see $[\mathrm{DO}]$.

There is nothing special about the $\Lambda_{i}$ functions used in the definition (4.1). If $L_{i}^{\prime}$ with $1 \leq i \leq 2 g$ is any basis for $\operatorname{Hom}_{0}(\Gamma, \mathbb{C})$ then there exist $\Lambda_{i}^{\prime}$ functions as in $(2.2)$ such that $L_{i}^{\prime}(\gamma)=\Lambda_{i}^{\prime}(\gamma z)-\Lambda_{i}^{\prime}(z)$ for all $\gamma$ in $\Gamma$. Also set $\Lambda_{0}^{\prime}=\Lambda_{0}=1$.

Proposition 4.1. For $f=\sum_{i=0}^{2 g} h_{i} \Lambda_{i}=\sum_{j=0}^{2 g} h_{j}^{\prime} \Lambda_{j}^{\prime}$ we have

$$
\sum_{i=0}^{2 g}\left(T_{n} h_{i}\right) \Lambda_{i}=\sum_{j=0}^{2 g}\left(T_{n} h_{j}^{\prime}\right) \Lambda_{j}^{\prime} .
$$

Proof. We express the linear dependence of $\Lambda_{j}^{\prime}$ and $\Lambda_{i}$ by writing

$$
\Lambda_{j}^{\prime}=\sum_{i} \alpha_{i j} \Lambda_{i}
$$

Thus

$$
f=\sum_{i=0}^{2 g} h_{i} \Lambda_{i}=\sum_{j=0}^{2 g} h_{j}^{\prime} \Lambda_{j}^{\prime}=\sum_{i, j=0}^{2 g} \alpha_{i j} h_{j}^{\prime} \Lambda_{i}
$$

so that $h_{i}-\sum_{j} \alpha_{i j} h_{j}^{\prime}=0$. Now

$$
\begin{aligned}
\sum_{i}\left(T_{n} h_{i}\right) \Lambda_{i}-\sum_{j}\left(T_{n} h_{j}^{\prime}\right) \Lambda_{j}^{\prime} & =\sum_{i}\left(T_{n} h_{i}\right) \Lambda_{i}-\sum_{i, j}\left(T_{n} h_{j}^{\prime}\right) \alpha_{i j} \Lambda_{i} \\
& =\sum_{i}\left(\left(T_{n} h_{i}\right)-\sum_{j} \alpha_{i j}\left(T_{n} h_{j}^{\prime}\right)\right) \Lambda_{i} \\
& =\sum_{i}\left(T_{n}\left(h_{i}-\sum_{j} \alpha_{i j} h_{j}^{\prime}\right)\right) \Lambda_{i}=0
\end{aligned}
$$

as required, completing the proof.

It is obvious that these Hecke operators $T_{n}((n, N)=1)$ inherit the multiplicativity properties of the usual Hecke operators. Furthermore, it is possible to give a simple characterization of the effect of $T_{p}$ 's ( $p$ prime not dividing $N$ ) on the Fourier coefficients of a holomorphic $f \in \widetilde{S}_{k}^{2}(N, v)$. 
Specifically, suppose that

$$
f(z)=\sum_{j} f_{j}(z) \int_{i \infty}^{z} g_{j}(w) d w
$$

for some $f_{j} \in S_{k}(N, v), g_{j} \in S_{2}(N)$. If

$$
f_{j}(z)=\sum_{m=1}^{\infty} a_{j}(m) e^{2 \pi i m z}, \quad g_{j}(z)=\sum_{m=1}^{\infty} c_{j}(m) e^{2 \pi i m z}
$$

then

$$
f(z)=\frac{1}{2 \pi i} \sum_{m}\left(\sum_{n} \frac{\sum_{j} a_{j}(m-n) c_{j}(n)}{n}\right) e^{2 \pi i m z} .
$$

Therefore, for every prime $p$ such that $(p, N)=1$,

$$
T_{p} f(z)=\frac{1}{2 \pi i} \sum_{m}\left(\sum_{n} \frac{\sum_{j} \widetilde{a}_{j}(m-n) c_{j}(n)}{n}\right) e^{2 \pi i m z}
$$

where $\widetilde{a}_{j}(l)=a_{j}(p l)+p^{k-1} a_{j}(l / p)\left(\right.$ with $a_{j}(\alpha):=0$ if $\left.\alpha \notin \mathbb{Z}\right)$ is the $l$ th Fourier coefficient of $T_{p} f_{j}(z)$.

This implies, in particular, that if $f$ is an eigenfunction of $T_{p}$ with eigenvalue $\lambda_{p}$, then

$$
\sum_{j} a_{j}(p) c_{j}(1)=\lambda_{p} \sum_{j} a_{j}(1) c_{j}(1) .
$$

It should finally be noted that in a similar manner we could construct operators induced by the Atkin-Lehner operators $U_{q}(q \mid N)$ for which an analogous discussion applies.

Acknowledgments. The authors wish to thank the referee for the very careful reading of the paper. The first named author would also like to thank the Max-Planck-Institut in Bonn for their support during the academic year 2000-2001 and Don Zagier for several enlightening conversations.

\section{References}

[CG] G. Chinta and D. Goldfeld, Grössencharakter L-functions of real quadratic fields twisted by modular symbols, Invent. Math. 144 (2001), 435-449.

[DO] N. Diamantis and C. O'Sullivan, Hecke theory of series formed with modular symbols and relations among convolution L-functions, Math. Ann. 318 (2000), 85-105.

[G1] D. Goldfeld, Zeta functions formed with modular symbols, Proc. Sympos. Pure Math. 66 (1999), 111-122.

[G2] - , The distribution of modular symbols, in: Number Theory in Progress, Proc. Intern. Conf. on Number Theory in Honor of A. Schinzel (Zakopane, 1997), Vol. 2, de Gruyter, 1999, 849-866.

[G3] -, Modular forms, elliptic curves and the ABC-conjecture, to appear. 
[GG] D. Goldfeld and P. Gunnells, Eisenstein series twisted by modular symbols for the group $G L_{n}$, Math. Res. Lett. 7 (2000), 747-756.

[I] H. Iwaniec, Topics in Classical Automorphic Forms, Grad. Stud. Math. 17, Amer. Math. Soc., Providence, RI, 1997.

[O] C. O'Sullivan, Properties of Eisenstein series formed with modular symbols, J. Reine Angew. Math. 518 (2000), 163-186.

[S] G. Shimura, Introduction to the Arithmetic Theory of Automorphic Functions, Publ. Math. Soc. Japan 11, Princeton Univ. Press, Princeton, NJ, 1971.

Department of Mathematics

Brown University

Providence, RI 02912, U.S.A.

E-mail: chinta@math.brown.edu

Department of Mathematics

Bronx Community College (CUNY)

University Ave. \& West 181 St.

Bronx, NY 10453, U.S.A.

E-mail: cormac@math.umd.edu
Department of Mathematics

McMaster University

Hamilton, Ontario

L8S 4K1, Canada

E-mail: diamant@icarus.mcmaster.ca

Received on 23.7.2001

and in revised form on 4.10.2001 